\title{
A New Preparation Method for Ophthalmic Drug Nanoparticles
}

Noriaki Nagai and Yoshimasa Ito*

Faculty of Pharmacy, Kinki University, 3-4-1 Kowakae, Higashi-Osaka, Osaka, 577-8502, Japan

\begin{abstract}
The most challenging task in ophthalmic therapy has long been the formulation of suitable ocular drug delivery systems due to the unique structure of the eye that restricts entry of the drug molecule at the site of action. Recently, the use of nanotechnology in the ophthalmic field has gained much attention, since nanoparticulate drug delivery is considered to be one of the most promising technologies to overcome poor drug stability and the difficulties in delivering drugs across biological barriers (improvement of bioavailability). This review demonstrates the usefulness of ophthalmic formulations containing drug nanoparticles. Furthermore, in this review, we introduce a new method established in our laboratory for the preparation of drug solid nanoparticles. This information provides significant information that can be used to design further studies aimed at developing less toxic eye drops.
\end{abstract}

Keywords: Nanoparticle; Ophthalmic formulations; Corneal toxicity; Transcorneal penetration; Drug delivery

\section{Introduction}

The eye is a small and complex organ that is separated from the rest of the body by multiple layers of biological barriers. Moreover, the internal ocular structures and tissues are protected from the external environment by the tight junctions of the corneal epithelium and the mucosal surface. The ophthalmic application of drugs is the primary route of administration for the treatment of various eye diseases, and is well-accepted by patients; however, in traditional formulations, only small amounts of the administered drug penetrate the cornea to reach the desired intraocular tissue due to corneal barriers and dilution caused by lacrimation [1-3]. Consequently, there is a need for the frequent instillation of concentrated solutions to obtain the desired therapeutic effect in both the anterior and posterior hemispheres of the eye. However, the frequent administration of drugs can cause corneal damage as well as undesirable side effects resulting from the systemic absorption of drugs through the nasolacrimal duct $[4,5]$. Therefore, the primary challenge for ocular drug delivery is how to circumvent these protective barriers in order to achieve therapeutically effective concentrations of drugs in the intraocular tissues. In this regard, it is very important to increase the effectiveness of drugs by enhancing their bioavailability [6]. In order to overcome these problems and increase ocular drug bioavailability, several strategies including the preparation of viscous solutions, micro/nanoparticles and hydrogels have been developed and investigated [1,4,7-10]. In the case of viscous solutions, numerous studies have demonstrated that they do not possess sufficient mechanical strength to resist the ocular clearance mechanism, and offer only a transient improvement in ocular residence time [11,12]. On the other hand, it has been reported that the capability of drugs to penetrate across the cornea can be significantly improved by decreasing the particle size using nanoparticles [3,9,13-15]. Ophthalmic formulations containing drug nanoparticles present a possible solution to the limitations surrounding ocular drug penetration [16-19], and it is known that decreasing direct cellular stimulation and reducing the amount drug used by increasing its bioavailability are useful ways to circumvent the side effects related to drug delivery [6]. It is expected that ophthalmic drug systems using nanoparticles may provide an alternative strategy for increasing ocular drug penetration [16-19]. This review addresses the usefulness of ophthalmic formulations containing drug nanoparticles. In addition, we introduce the new method for the preparation of drug solid nanoparticles.

\section{Design Considerations for Ocular Drug Nanomaterials}

The size of a particle influences its functionality in terms of its uptake, residence in circulation, adherence, degradation, as well as clearance [20-24]. The fate of particles inside the body has been reported as follows: $\geq 2 \mu \mathrm{m}$, trapped inside liver cells; $\geq 300-400 \mathrm{~nm}$, captured by macrophages and excreted; $\geq 200 \mathrm{~nm}$, filtered in the spleen; $\geq 100 \mathrm{~nm}$, escape from blood vessels through the endothelial lining. Thus, size governs the movement of nanoparticles inside tissues. In the ophthalmic field, nanoparticles in sizes ranging from 10 to 1000 $\mathrm{nm}$ allow for the improved topical passage of large, water insoluble molecules through the barriers of the ocular system [25]. Superficial barriers impede direct and systemic drug access to the specific site of action. Drug loaded nanoparticles show favorable biological properties including prolonged residence time for eye drops, decreased toxicity, and increased ability of the drug to penetrate into the deeper layers of the ocular structure and aqueous humor thus minimizing precorneal drug loss caused by rapid tear fluid turnover $[6,26]$. Techniques were planned to transform nanoparticles from lipophilic to hydrophilic and to down-regulate irritation to the eye. Preparations that include nanoparticles could be very useful for the extended delivery of ophthalmic drugs $[2,27,28]$. Preparations that include nanoparticles have been used to deliver ocular drugs to target sites in the treatment of many eye diseases as summarized in Table $1[13,29]$. An ideal ocular drug delivery system should possess key properties that include: (I) a controlled and sustained release profile to maintain a therapeutic concentration of the drug over a prolonged period of time to reduce the frequency of administration; (II) specific targeting and prolonged retention in the diseased tissues to improve therapeutic efficiency and mitigate side effects; and (III) patient-friendly delivery routes that minimize or eliminate side effects resulting directly from these administration methods. At present, nanocarrier-based ocular drug delivery systems appear to be the most promising tool to meet the

*Corresponding author: Yoshimasa Ito, $\mathrm{PhD}$, Faculty of Pharmacy, Kinki University, 3-4-1 Kowakae, Higashi-Osaka, Osaka 577-8502 (Japan), Tel: +81 6 4307 3640; Fax: +81 66730 1394; E-mail: itoyoshi@phar.kindai.ac.jp

Received March 18, 2014; Accepted July 30, 2014; Published August 07, 2014

Citation: Nagai N, Ito Y (2014) A New Preparation Method for Ophthalmic Drug Nanoparticles. Pharm Anal Acta 5: 305. doi:10.4172/2153-2435.1000305

Copyright: (C) 2014 Nagai N, et al. This is an open-access article distributed under the terms of the Creative Commons Attribution License, which permits unrestricted use, distribution, and reproduction in any medium, provided the original author and source are credited. 
primary requirements of an ideal ocular delivery system.

\section{Preparation of Ocular Drug Nanomaterials}

Previous research over several decades focused on two major approaches to the design of nanocarriers: bottom-up synthesis and a top-down approach. Bottom-up synthesis, which is based on self assembly and emulsion systems, has been studied extensively in the past, and a variety of potential nanocarriers have been developed using this method, for example, polymeric nanoparticles, micelles, liposomes, nanoemulsions, dendrimers, biodegradable and non-biodegradable carriers, solid lipid nanoparticles, magnetic nanoparticles etc. A majority of these carriers are colloidal systems governed by different forces such as hydrophobic interactions, Van der Waals forces, hydrogen bonding, and ionic interactions. With this approach, high polydispersities are often exhibited, and the systems developed sometimes present certain limitations. In the in vivo drug release profiles, the physicochemical characteristics and degradation kinetics of these carriers are difficult to evaluate and reproduce as they are variable. On the other hand, major

\begin{tabular}{|l|l|}
\hline \multicolumn{1}{|c|}{ Eye disease } & \multicolumn{1}{c|}{ Drug nanoparticles } \\
\hline Corneal Disease & $\begin{array}{l}\text { IM-CS-NPs, CS-CsA-SLN, DS-NS, CS/ } \\
\text { TCS-SA NPs }\end{array}$ \\
\hline Keratoplasty & FK506 PLGA \\
\hline Corneal neovascularization & Flt23k NPs, Pluronic F 127 diacrylate \\
\hline Viral keratitis & SLNs, NLCs \\
\hline Wounds of the corneal epithelium & All-trans retinoic acid NPs \\
\hline Corneal gene therapy & Chitosan-DNA NPs, deliver plasmid \\
\hline Corneal tissue engineering & Nanofibrous tissue-engineered scaffolds \\
\hline Glaucoma & HDNP, CS-HA-NPs, (SLN)NPs \\
\hline Uveitis & PEG-block-PLA, NP-PEG-TAM \\
\hline Retinal diseases & Aerosolized NPs, \\
& $\begin{array}{l}\text { Vacancy engineered mixed-valence-state } \\
\text { cerium oxide NPs }\end{array}$ \\
\hline Retinal gene therapy & $\begin{array}{l}\text { VMD2-eGFP, PEG-POD NPs, Compacted } \\
\text { DNA NPs }\end{array}$ \\
\hline Age-related macular degeneration & PEG-LPH-NP siRNA, PLA/PLA-PEO NPs \\
\hline Choroidal neovascularization & $\begin{array}{l}\text { bFGF-NPs, (CK30PEG)-compacted DNA } \\
\text { NPs }\end{array}$ \\
\hline
\end{tabular}

CS, chitosan; CsA, cyclosporine A; HA, Hyaluronic acid; HDNP, hybrid dendrimer nanoparticle; IM, indomethacin; LPH, liposomeprotamine-hyaluronic acid; LC, lipid carrier; NPs, Nanoparticles; NS, nanoparticle suspensions; PEG, poly (ethylene) glycol; PEO, polyethylene oxide; PLA, poly (lactic acid); PLGA, poly (lactic-coglycolic acid); SA, sodium alginate; SL, solid lipid; TAM, tamoxifen; TCS, thiolated chitosan; VMD2-eGFP, retinal pigment epithelium-specific reporter vector; SLN solid lipid nanoparticles;

Table 1: Drug nanoparticles in the ophthalmic field. advancements have been made recently in the fabrication technology by the introduction of the "top-down" approach for micro and nanofabrication systems using electromechanical techniques. This approach exhibits the potential for designing nanoparticles with precision in terms of particle shape and size. Such an approach can provide control over particle size, functionality, and precise particle geometry. This approach may also resolve the limitations of the bottom-up approach in research.

We have also designed ophthalmic formulations containing drug nanoparticles obtained by mill methods [30,31]. Ophthalmic solutions containing indomethacin (IMC) solid nanoparticles (IMC ${ }_{\text {nano }}$ ) were prepared using zirconia beads and Bead Smash 12 (a bead mill, Wakenyaku Co. Ltd, Kyoto, Japan) (Figure 1). Briefly, zirconia beads (diameter: $2 \mathrm{~mm}$ ) were added to IMC microparticles (solid) containing BAC, mannitol or methylcellulose (MC), and the mixtures were crushed with the bead mill for $30 \mathrm{sec}\left(3,000 \mathrm{rpm}, 4^{\circ} \mathrm{C}\right)$. The mixtures were dispersed in saline with or without $5 \% 2$-Hydroxypropyl- $\beta$ cyclodextrin (HP $\beta C D)$, and crushed again with the bead mill $(5,500$ $\mathrm{rpm}, 30 \mathrm{sec} \times 15$ times, $4^{\circ} \mathrm{C}$ ) using smaller zirconia beads (diameter: 0.1 $\mathrm{mm}$ ). The compositions of the dispersions containing IMC are shown in Table 2. $0.5 \%$ IMC is equivalent to $14.0 \mathrm{mM} \mathrm{IMC}$; the $\mathrm{pH}$ was 6.5 for both ophthalmic dispersions containing IMC micro- or nanoparticles. The IMC particle size reached the nano order by the bead mill method using IMC microparticles containing BAC, mannitol, $\mathrm{HP} \beta \mathrm{CD}$ and $\mathrm{MC}$ (IMC nanoophthalmic solution, (Figure 2), particle size $76 \pm 59 \mathrm{~nm}$, mean \pm S.D.). In addition, noprecipitation in the dispersions containing IMC nanoparticles was observed 7 days after preparation. Furthermore, the IMC ${ }_{\text {nano }}$ preparations showed high antimicrobial activity approximately equal to that of a $0.001 \% \mathrm{BAC}$ solution, a preservative used in the ophthalmic field.

\section{Usefulness of Ophthalmic Formulations containing Drug Solid Nanoparticles obtained by Bead Mill Methods}

It is very important to elucidate the corneal toxicity and permeability of ophthalmic solutions containingIMC solid nanoparticles $\left(\mathrm{IMC}_{\mathrm{nano}}\right.$ ). Therefore, we evaluated the transcorneal penetration of IMC ${ }_{\text {nan }}$ and its effects on corneal damage using human corneal epithelial cells (HCE-T), and rat and rabbit corneas [30]. The corneal wounds of rat eyes instilled with commercially available IMC eye drops (INDOMELOL ${ }^{\circledR}$ eye drops, Senju Pharmaceutical Co., Ltd, Osaka, Japan) showed 21.6\% healing while those instilled with $\mathrm{IMC}_{\text {nano }}$ showed $48.1 \%$ healing $12 \mathrm{hr}$ after corneal epithelial abrasion. In addition, the viability of HCE-T cells

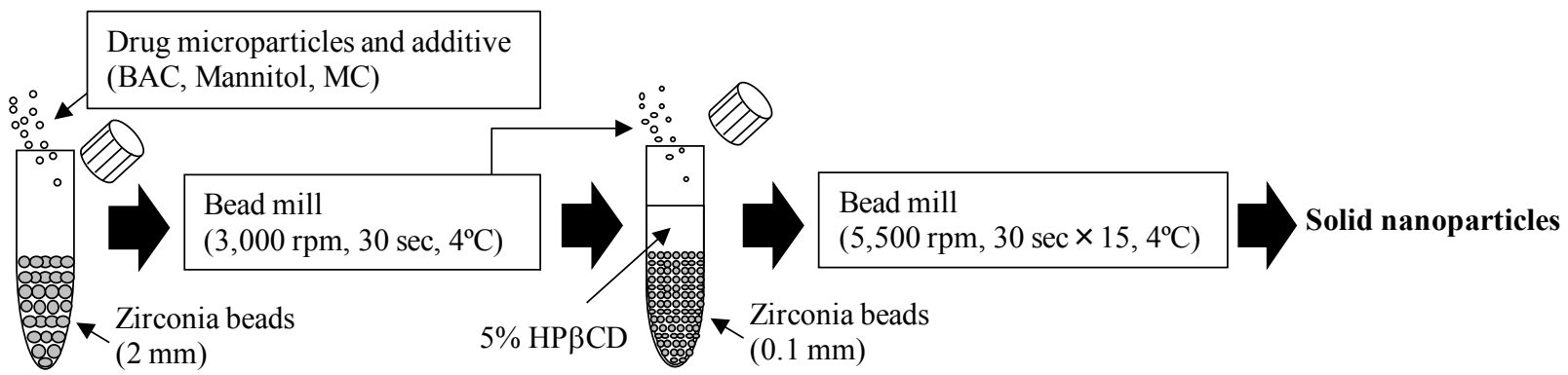

BAC, benzalkonium chloride; HP $\beta C D$, 2-hydroxypropyl- $\beta$-cyclodextrin; MC, methyl cellulose

Figure 1: Preparation procedures for ophthalmic drug solid nanoparticles by the mill method. 
Citation: Nagai N, Ito Y (2014) A New Preparation Method for Ophthalmic Drug Nanoparticles. Pharm Anal Acta 5: 305. doi:10.4172/21532435.1000305

Page 3 of 4

\begin{tabular}{|l|c|c|c|c|c|c|}
\hline \multirow{2}{*}{ Formulation } & \multicolumn{5}{|c|}{ Content $(\mathbf{w} / \mathbf{v} \%)$} & Treatment \\
\cline { 2 - 7 } & IMC microparticles & BAC & D-Mannitol & HP $\beta$ CD & 0.5 & Bead mill \\
\hline IMC $_{\text {nano }}$ & 0.5 & 0.001 & 0.1 & 5.0 & 0.5 & (n) \\
\hline
\end{tabular}
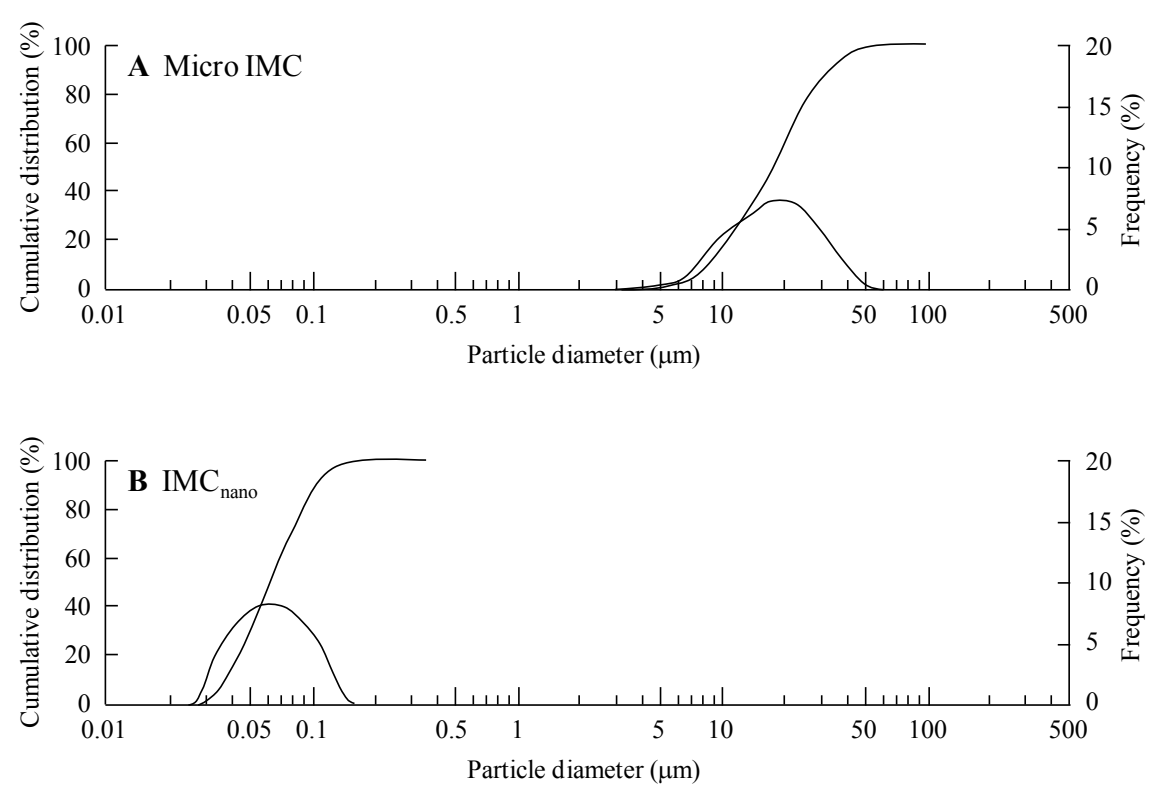

Figure 2: Cumulative size distribution and frequency of $0.5 \%$ IMC solid nanoparticles. IMC solid nanoparticleswere prepared as outlined in Figure 1. Particle size was determined using a nanoparticle size analyzer SALD-7100 (refractive index 1.60$0.10 \mathrm{i})$. Particle size: micro IMC, $17.2 \pm 12.8 \mu \mathrm{m}$; IMCnano, $76 \pm 59 \mathrm{~nm}$ (mean \pm S.D.).

treated with IMC $\mathrm{nano}_{\text {o }}$ was significantly higher than that of cells treated with commercially available IMC, pranoprofen (NIFLAN ${ }^{\circledR}$ eye drops, Senju Pharmaceutical Co., Ltd, Osaka, Japan), diclofenac (DICLOD ${ }^{\circledR}$ eye drops, WAKAMOTO Co., Ltd., Tokyo, Japan), bromfenac (BRONUK ${ }^{\circledR}$ eye drops, Senju Pharmaceutical Co., Ltd, Osaka, Japan) or nepafenac (NEVANAC ${ }^{\circledR}$ eye drops, ALCON Japan Ltd., Tokyo, Japan), and the accumulation of IMC in HCE-T cells treated with $\mathrm{IMC}_{\text {nano }}$ was less than that in cells treated with commercially available IMC. On the other hand, the penetration of IMC from IMC ${ }_{\text {nano }}$ was higher than from commercially available IMC eye drops in HCE-T cell monolayers. Furthermore, we have demonstrated the corneal penetration of ophthalmic solutions containing solid nanoparticles using rabbit corneas, which include corneal epithelial, stromal and endothelial cells (in vitro and in vivo transcorneal penetration experiments). In the in vitro study, the penetration coefficients through the cornea and the cornea/preparation partition coefficients were higher, and the diffusion constants within the cornea lower than those for commercially available IMC eye drops. The IMC penetration rate from $\mathrm{IMC}_{\text {nano }}\left(18.9 \pm 1.95 \mathrm{nmol} / \mathrm{cm}^{2} / \mathrm{h}, \mathrm{n}=5\right)$ was significantly higher in comparison with commercially available IMC eye drops $(5.43 \pm 2.59$ $\mathrm{nmol} / \mathrm{cm}^{2} / \mathrm{h}, \mathrm{n}=5$ ). In the in vivo study, noIMC from commercially available IMC eye drops was detected in the aqueous humor until 50 min after administration; however, the lag time from $\mathrm{IMC}_{\text {nano }}$ eye drops was ca. $25 \mathrm{~min}$. The penetration rate from IMC $\mathrm{Imas}_{\text {nano }}$ higher than those from commercially available IMC eye drops, and the penetration rate from $\mathrm{IMC}_{\text {nano }}$ and the area under the IMC concentration-time curve $\left(A U C_{\mathrm{IMC}}\right)$ were approximately 10.7 times greater in comparison with commercially available IMC eye drops. These results show that nanoparticle formulations provide a reduction in corneal toxicity, and may make it possible to decrease the amount of drug used via an increase in bioavailability, thus resulting in a reduction in drug toxicity.

\section{Conclusion}

In this review, we introduce the applications of nanoparticles in the ophthalmic field. Future work should focus on investigating combinations of additives to enhance the favorable properties of nanoparticles. It will be interesting to observe what benefits these innovative drug preparations can provide to patients. In addition, we describe a new method for preparing drug solid nanoparticles established by us [30-32].The particle size of our drug solid nanoparticles is of high quality (particle size, approximately 60-80 nm). Dispersions containing these drug nanoparticles are tolerated better by human and rat corneal epithelial cells than commercially available eye drops, since the accumulation of drug in nanoparticles is lower than from solutions. Furthermore, the state of the dispersions containing drug solid nanoparticles does not affect the antimicrobial activity of BAC against E. coli, and thecorneal penetration of drug solid nanoparticles is significantly better than that from commercially available eye drops. The cost-effectiveness of the new preparation method is also very

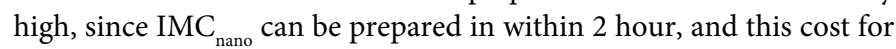
preparation is only reagent cost. It is expected that ocular drug delivery systems using drug nanoparticles may expand their usage for therapy in the ophthalmologic field.

\section{References}

1. Diebold Y, Jarrín M, Sáez V, Carvalho EL, Orea M, et al. (2007) Ocular drug delivery by liposome-chitosan nanoparticle complexes (LCS-NP). See comment in PubMed Commons below Biomaterials 28: 1553-1564.

2. Baba K, Tanaka Y, Kubota A, Kasai H, Yokokura S, et al. (2011) A method for enhancing the ocular penetration of eye drops using nanoparticles of hydrolyzable dye. See comment in PubMed Commons below J Control Release 153: 278-287. 
Citation: Nagai N, Ito Y (2014) A New Preparation Method for Ophthalmic Drug Nanoparticles. Pharm Anal Acta 5: 305. doi:10.4172/21532435.1000305

3. Li X, Zhang Z, Li J, Sun S, Weng Y, et al. (2012) Diclofenac/biodegradable polymer micelles for ocular applications. See comment in PubMed Commons below Nanoscale 4: 4667-4673.

4. Asasutjarit R, Thanasanchokpibull S, Fuongfuchat A, Veeranondha S (2011) Optimization and evaluation of thermoresponsive diclofenac sodium ophthalmic in situ gels. See comment in PubMed Commons below Int J Pharm 411: 128 135.

5. Mahmoud AA, El-Feky GS, Kamel R, Awad GE (2011) Chitosan/sulfobutylether$\hat{I}^{2}$-cyclodextrin nanoparticles as a potential approach for ocular drug delivery. See comment in PubMed Commons below Int J Pharm 413: 229-236.

6. Ammar HO, Salama HA, Ghorab M, Mahmoud AA (2009) Nanoemulsion as a potential ophthalmic delivery system for dorzolamide hydrochloride. See comment in PubMed Commons below AAPS PharmSciTech 10: 808-819.

7. El-Kamel $\mathrm{AH}$ (2002) In vitro and in vivo evaluation of Pluronic F127-based ocular delivery system for timolol maleate. See comment in PubMed Commons below Int J Pharm 241: 47-55.

8. Sultana Y, Aqil M, Ali A (2006) Ion-activated, Gelrite-based in situ ophthalmic gels of pefloxacin mesylate: comparison with conventional eye drops. See comment in PubMed Commons below Drug Deliv 13: 215-219.

9. Gupta H, Aqil M, Khar RK, Ali A, Bhatnagar A, et al. (2011) Biodegradable levofloxacin nanoparticles for sustained ocular drug delivery. See comment in PubMed Commons below J Drug Target 19: 409-417.

10. Casolaro M, Casolaro I, Lamponi S (2012) Stimuli-responsive hydrogels for controlled pilocarpine ocular delivery. See comment in PubMed Commons below Eur J Pharm Biopharm 80: 553-561.

11. Davies NM, Farr SJ, Hadgraft J, Kellaway IW (1991) Evaluation of mucoadhesive polymers in ocular drug delivery. I. Viscous solutions. See comment in PubMed Commons below Pharm Res 8: 1039-1043.

12. Liu S, Jones L, Gu FX (2012) Nanomaterials for ocular drug delivery. See comment in PubMed Commons below Macromol Biosci 12: 608-620.

13. Rahul M, Mohita U, Sanat M (2014) Design Considerations for Chemotherapeutic Drug Nanocarriers. Pharm Anal Acta 5: 279 Review.

14. Zhou HY, Hao JL, Wang S, Zheng Y, Zhang WS (2013) Nanoparticles in the ocular drug delivery. See comment in PubMed Commons below Int $J$ Ophthalmol 6: 390-396.

15. Rafie F, Javadzadeh $Y$, Javadzadeh AR, Ghavidel LA, Jafari B, et al. (2010) In vivo evaluation of novel nanoparticles containing dexamethasone for ocular drug delivery on rabbit eye. See comment in PubMed Commons below Curr Eye Res 35: 1081-1089.

16. Cohen S, Yoshioka T, Lucarelli M, Hwang LH, Langer R (1991) Controlled delivery systems for proteins based on poly(lactic/glycolic acid) microspheres. See comment in PubMed Commons below Pharm Res 8: 713-720.

17. Tomoda K, Watanabe A, Suzuki K, Inagi T, Terada H, et al. (2012) Enhanced transdermal permeability of estradiol using combination of PLGA nanoparticles system and iontophoresis. See comment in PubMed Commons below Colloids Surf B Biointerfaces 97: 84-89.

18. Tomoda K, Terashima H, Suzuki K, Inagi T, Terada H, et al. (2012) Enhanced transdermal delivery of indomethacin using combination of PLGA nanoparticles and iontophoresis in vivo. See comment in PubMed Commons below Colloids Surf B Biointerfaces 92: 50-54.

19. Tomoda K, Terashima H, Suzuki K, Inagi T, Terada H, et al. (2011) Enhanced transdermal delivery of indomethacin-loaded PLGA nanoparticles by iontophoresis. See comment in PubMed Commons below Colloids Surf B Biointerfaces 88: 706-710.

20. Begley DJ (2004) Delivery of therapeutic agents to the central nervous system: the problems and the possibilities. See comment in PubMed Commons below Pharmacol Ther 104: 29-45.
21. Bareford LM, Swaan PW (2007) Endocytic mechanisms for targeted drug delivery. See comment in PubMed Commons below Adv Drug Deliv Rev 59 748-758.

22. Dobrovolskaia MA, Aggarwal P, Hall JB, McNeil SE (2008) Preclinical studies to understand nanoparticle interaction with the immune system and its potential effects on nanoparticle biodistribution. See comment in PubMed Commons below Mol Pharm 5: 487-495.

23. Chouly C, Pouliquen D, Lucet I, Jeune JJ, Jallet P (1996) Development of superparamagnetic nanoparticles for MRI: effect of particle size, charge and surface nature on biodistribution. See comment in PubMed Commons below $J$ Microencapsul 13: 245-255.

24. Toy R, Peiris PM, Ghaghada KB, Karathanasis E (2014) Shaping cance nanomedicine: the effect of particle shape on the in vivo journey of nanoparticles. See comment in PubMed Commons below Nanomedicine (Lond) 9: 121-134.

25. Diebold Y, Calonge M (2010) Applications of nanoparticles in ophthalmology See comment in PubMed Commons below Prog Retin Eye Res 29: 596-609.

26. Kesavan K, Balasubramaniam J, Kant S, Singh PN, Pandit JK (2011) Newe approaches for optimal bioavailability of ocularly delivered drugs: review. See comment in PubMed Commons below Curr Drug Deliv 8: 172-193.

27. Maiti S, Paul S, Mondol R, Ray S, Sa B (2011) Nanovesicular formulation of brimonidine tartrate for the management of glaucoma: in vitro and in vivo evaluation. See comment in PubMed Commons below AAPS PharmSciTech 12: $755-763$

28. Jung HJ, Chauhan A (2012) Temperature sensitive contact lenses for triggered ophthalmic drug delivery. See comment in PubMed Commons below Biomaterials 33: 2289-2300.

29. Zarbin MA, Montemagno C, Leary JF, Ritch R (2010) Nanotechnology in ophthalmology. See comment in PubMed Commons below Can J Ophthalmo 45: $457-476$

30. Nagai $N$, Ito $Y$, Okamoto $N$, Shimomura $Y$ (2014) A nanoparticle formulation reduces the corneal toxicity of indomethacin eye drops and enhances its corneal permeability. See comment in PubMed Commons below Toxicology 319: 53-62.

31. Nagai $N$, Ono $H$, Hashino $M$, Ito $Y$, Okamoto $N$, et al. (2014) Improved corneal toxicity and permeability of tranilast by the preparation of ophthalmic formulations containing its nanoparticles. See comment in PubMed Commons below J Oleo Sci 63: 177-186.

32. Nagai N, Ito $Y(2014)$ Therapeutic effects of gel ointments containing tranilast nanoparticles on paw edema in adjuvant-induced arthritis rats. See comment in PubMed Commons below Biol Pharm Bull 37: 96-104. 